\title{
Tailoring the interfacial magnetic anisotropy in multiferroic field-effect devices
}

\author{
Daniele Preziosi, ${ }^{*}$ Ignasi Fina, Eckhard Pippel, and Dietrich Hesse \\ Max Planck Institute of Microstructure Physics D-06120 Halle, Germany \\ Xavi Marti \\ Centre d'Investigacions en Nanociencia I Nanotechnologia (CIN2), CSIC-ICN Bellaterra 08193, Barcelona, Spain \\ Francis Bern and Michael Ziese \\ Institute for Experimental Physics II, University of Leipzig Linnèstrasse 5, 04103 Leipzig, Germany \\ Marin Alexe \\ Department of Physics, University of Warwick Coventry CV4 7AL, UK \\ (Received 30 July 2014; revised manuscript received 1 September 2014; published 29 September 2014)
}

\begin{abstract}
Ferroelectric field-effect devices based on perovskite oxide materials offer a new possibility to exploit emergent interfacial effects such as the electrostatic modification of the transport and magnetic properties of strongly correlated materials and to prove the magneto-electric coupling at the interface between the two different ferroic materials. Here we report on the reversible modulation of the interfacial magnetic and magnetotransport properties of $\mathrm{La}_{0.825} \mathrm{Sr}_{0.175} \mathrm{MnO}_{3}$ thin films induced by switching the ferroelectric polarization of a top $\mathrm{PbZr}_{0.2} \mathrm{Ti}_{0.8} \mathrm{O}_{3}$ layer. Anisotropic magnetoresistance (AMR) measurements were performed applying a magnetic field $H$ in a plane perpendicular to the current density. By rotating $H$ from the out-of-plane towards the in-plane direction, upon the ferroelectric polarization switching, a modulation of the normalized AMR amplitude was achieved. The dynamical electrostatic coupling at the interface of the two oxides is responsible for a reconstruction of the Mn $3 d e_{g}$ orbitals which in turn affects the surface magnetic anisotropy of the magneto-electric system. The present work might have a broader impact, including in the field of multiferroic tunnel junctions, due to a better understanding of the coupling at the interface of the two ferroic oxides where the influence of the polarization on the magnetic degree of freedom is accomplished.
\end{abstract}

DOI: 10.1103/PhysRevB.90.125155

PACS number(s): 75.30.Gw, 81.15.Fg, 73.63.-b

\section{INTRODUCTION}

Over the past few decades, strongly correlated materials (SCMs) have been attracting a lot of interest because of their appealing electronic properties [1]. The discovery of the unusually large change of the transport properties under external magnetic field, known as colossal magnetoresistance [2] (CMR), has been a milestone in material science. The fingerprint of some SCMs [3] is the interplay between charge, spin, lattice, and orbital ordering, which in turn leads to a remarkable sensitivity of the system to external stimuli [4]. Since the seminal studies carried out by Jonker and van Santen [5], one of the most studied SCMs is strontium-doped lanthanum manganite, i.e., $\mathrm{La}_{1-x} \mathrm{Sr}_{x} \mathrm{MnO}_{3}$ (LSMO). In the LSMO, the electronic correlations emerge in a manifold of ground states usually summarized in a complex and rich phase diagram [6]. In particular, the interplay between the transport and magnetic properties of LSMO, modulated by the application of an external electric field, is of great interest to basic physics as well as from a technological point of view. Ferroelectric field-effect devices (FeFEDs) in thin-film form, where the LSMO is gated by an adjacent ferroelectric oxide, allow for the characterization of the transport and magnetic properties of LSMO under the application of an electric field. Tunable interfacial accumulation and depletion states can be

\footnotetext{
*preziosi@mpi-halle.mpg.de
}

realized upon ferroelectric switching, which, in turn, affect the transport and magnetic properties of the LSMO. As a matter of fact, it has been already shown that the transport and magnetic properties of LSMO $(x=0.2)$ can be tuned by the reversal of the ferroelectric polarization $P$ owing to the intimate relation between the valence state and the magnetic moment of the manganese ions [7-9]. AMR measurements can reveal interesting magnetic properties, and the related microscopic mechanism is at the base of the most modern concept of spintronics. Indeed, in AMR, the variation of the resistivity depends on the orientation of the magnetization $M$ with respect to both the current density $J$ and the crystal axes. From the angular dependence of the AMR [10,11], information on the spin-orbit coupling (SOC) [12] and the magneto-elastic coupling (Jahn-Teller distortions) can be obtained. However, up to now, reports about the possibility to electrostatically modulate the AMR amplitude have not been presented. Here, we report on magnetic and anisotropic magnetotransport measurements in fully epitaxial $\mathrm{PbZr}_{0.2} \mathrm{Ti}_{0.8} \mathrm{O}_{3}(\mathrm{PZT}) / \mathrm{LSMO} / \mathrm{STO}$ FeFEDs with a Sr content in the LSMO of $x=0.175$, which is at the boundary between insulating and metallic phases [13]. First, we show that, upon the ferroelectric switching of the PZT layer, the magnetic properties of the LSMO can be favorably modulated. Then, an interfacial tetragonal-like $c / a$ ratio modulation of the LSMO layer, induced and triggered by the ferroelectric switching, is proposed to account for the observed intriguing dependence of the normalized AMR amplitude. 


\section{EXPERIMENT}

LSMO/PZT heterostructures were grown onto STO and LSAT single crystals via pulsed laser deposition (PLD), by ablating the respective stoichiometric ceramic targets with a $\mathrm{KrF}$ excimer laser $(\lambda=248 \mathrm{~nm})$. The deposition was performed at a substrate temperature of $600{ }^{\circ} \mathrm{C}$ under an $\mathrm{O}_{2}$ partial pressure of 0.2 and 0.28 mbar for the LSMO and PZT, respectively. All the samples were gradually cooled to room temperature in an $\mathrm{O}_{2}$ atmosphere of $\sim 100$ mbar.

Magnetization measurements were carried out in a magnetic property measurement system (MPMS-7, Quantum Design) in dc mode with a commercial sample holder equipped with wires to allow the in situ switching of the ferroelectric polarization of the top PZT layer (see Fig. S3).

Magnetotransport measurements were performed by using the physical properties measurement system (PPMS) of Quantum Design using a standard sample rotator.

The two FeFEDs employed for the magnetic and transport measurements were grown with the same LSMO $(5 \mathrm{~nm})$ and PZT $(170 \mathrm{~nm})$ thicknesses. A few samples were grown with 30-nm-thick PZT layers.

\section{RESULTS}

Here we present two types of investigations carried out in order to reveal the coupling between polarization and magnetic order at the LSMO/PZT interface: one performing magnetization measurements and one magnetotransport measurements, both using special designed FeFEDs which allowed an in situ ferroelectric switching.

Figure 1 summarizes the structural and morphological characterization of the PZT/LSMO/STO heterostructures. In Fig. 1(a), a high-angle annular dark field (HAADF) scanning transmission electron microscopy (STEM) image of the PZT/LSMO/STO system is shown, revealing the epitaxial
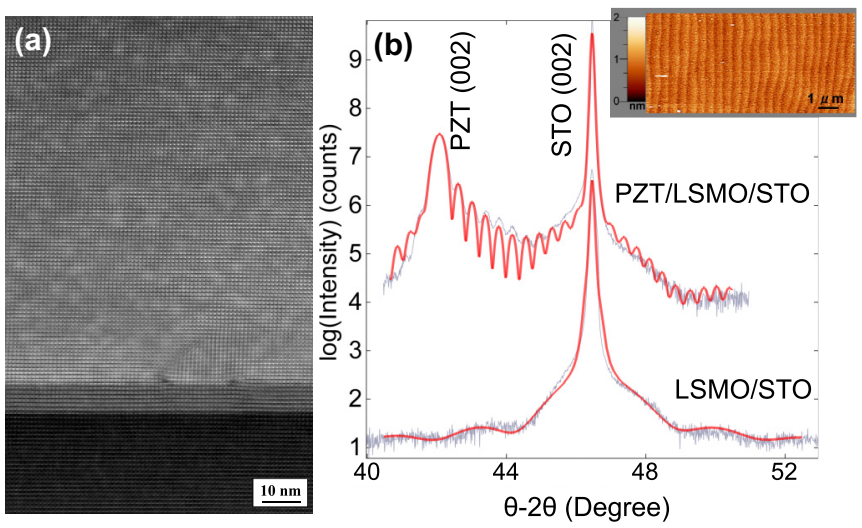

FIG. 1. (Color online) Structural and morphological characterization of the LSMO/PZT/STO heterostructures. In panel (a), the HAADF-STEM image shows 5-nm- and 170-nm-thick layers of LSMO and PZT, respectively. (b) X-ray diffraction patterns (gray lines) of a PZT/LSMO/STO unpatterned bilayer (upper curve) and of a LSMO/STO single layer (bottom curve) with simulated fitting curves (red curves) giving a thickness of 5 and $30 \mathrm{~nm}$ for the LSMO and the PZT layer, respectively. (Inset) AFM-topography image of the 30-nm-thick PZT. and coherent growth of both oxide materials. Although misfit dislocations are visible at the PZT/LSMO interface, EDX maps taken across them show that the interface is still sharp and that no sizable lead diffusion occurred into the underneath LSMO layer (see Figs. S1 and S2 of the Supplementary Information [14]). The inset of Fig. 1(b) shows an AFM image of the PZT/LSMO/STO bilayer, which reveals a flat surface with a root mean square $(\mathrm{rms})$ roughness of $0.335 \mathrm{~nm}$; the presence of the terrace structure indicates a layer-by-layer growth of the PZT layer, demonstrating the good optimization of the growth parameters. Finally, Fig. 1(b) shows the $\theta-2 \theta$ diffraction patterns acquired for a PZT (30 nm)/LSMO (5 $\mathrm{nm}$ ) bilayer (upper curve), and a LSMO (5 nm) single layer (lower curve). The red solid curves represent a fit made in the framework of the fully dynamical diffraction theory [15]. A good match between the measured oscillations around the (002) STO peak and the fitting curve occurs when the out-of-plane parameter of the LSMO layer is chosen equal to $(0.391 \pm 0.001) \mathrm{nm}$. Accordingly, the unit cell of the 5-nm-thick LSMO film can be indexed in the pseudocubic notation with the same lattice parameter of the STO substrate, i.e., $a=0.3905 \mathrm{~nm}$. The x-ray diffraction pattern also shows a fully $c$-axis oriented PZT film $(c=$ $0.429 \mathrm{~nm}$ ), and the observed Kiessig fringes corroborate the TEM data, revealing the high crystalline quality of the oxide materials.

Figure 2 shows the results obtained from the in situ $P$ dependent magnetometer experiments. Accordingly, Fig. 2(a) shows a schematic view of the FeFED that was used. Large-area capacitors $\left(0.64 \mathrm{~mm}^{2}\right.$ area each $)$ were fabricated evaporating top Au electrodes using a shadow mask directly on top of the PZT/LSMO/STO heterostructure. Top electrodes of all functioning capacitors were contacted in parallel in groups

(a)
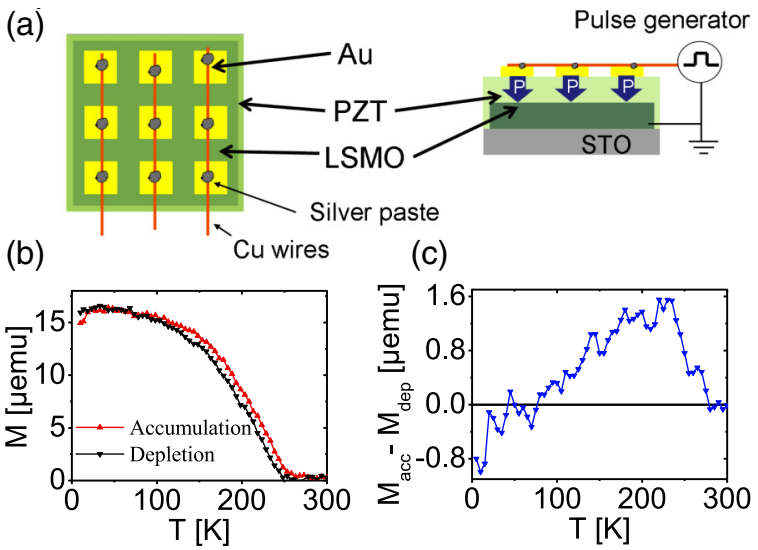

FIG. 2. (Color online) (a) Schematic view of the FeFED used for the superconducting quantum interference device measurements by in situ ferroelectric switching. The Au/PZT/LSMO/STO capacitors were contacted in groups of three by using simple $\mathrm{Cu}$ wires and silver paste, as schematically shown in the top view (left). The switching was performed by applying short voltage pulses and measuring the switching current, as shown in Fig. S3 of Supplementary Information [14]. (b) Temperature dependence of the magnetization for both polarization states acquired in field cooling with $H=0.01 \mathrm{~T}$. (c) Calculated difference between the magnetization values obtained in accumulation and depletion states, respectively. 
of three after an initial screening. This allowed fabrication of a rather macroscopic sample and ferroelectric switching of a total area of $5.76 \mathrm{~mm}^{2}$, as sketched in the cross section of Fig. 2(a). Upon application of a $5 \mathrm{~V}$ pulse the PZT polarization can be switched from pointing down [toward the LSMO, as sketched in Fig. 2(a)] to up (away from the LSMO). Figure 2(b) shows the magnetization measured in field cooling $(H=10 \mathrm{mT})$ as a function of temperature for down and up polarization states. It is worth noting that polarization pointing down leads to a depletion state (down triangle) and polarization pointing up leads to an accumulation state (up triangle). As a result, a modulation of the LSMO Curie temperature $T_{\mathrm{C}}$, from 248 to $263 \mathrm{~K}$, as well as of the magnetization $M$ upon the ferroelectric switching, is clearly observed. Theoretical studies, undertaken on similar complex oxide heterostructures, confirmed that $P$ switching, inducing an electrostatic depletion or accumulation of carriers at the interface between the two oxides, produces an alteration of the interfacial spin [16] and/or orbital $[17,18]$ configurations inherent to the interfacial Mn ions, which in turn is capable to modulate the magnetic properties of the system. Moreover, a similar $T_{\mathrm{C}}$ modulation by switching the LSMO layer from the depletion to the accumulation state was previously reported [8,19], but here it occurs at a much higher temperature. As a result, Fig. 2(c) shows the difference between the magnitude values of $M$, as function of temperature, measured in accumulation and depletion. This difference is positive from $270 \mathrm{~K}$ until 50 $\mathrm{K}$ where the sign reverses. At almost $230 \mathrm{~K}$ a maximum magnetization modulation of $1.5 \times 10^{-6} \mathrm{emu}$ is obtained. This corresponds to a variation of about $0.34 \mu_{B} / \mathrm{Mn}$ induced by $P$ switching.

Figure 3 summarizes the results obtained from the magnetotransport characterization of the LSMO/PZT system. In this respect, the related FeFED was engineered using $\mathrm{AlO}_{x^{-}}$ masked substrates in order to allow an in situ growth of the LSMO and PZT oxide materials in a prepatterned Hall bar (HB) geometry. On top of the HB channel [see Fig. 3(a)], a $\mathrm{Cu}$ top electrode was evaporated and subsequently suitably patterned by using a wet-etching procedure. The resulting FeFED enabled in situ $P$ switching. The standard ferroelectric hysteresis loop of the device revealed, as expected from high-quality PZT films [20], a saturation polarization value of almost $100 \mu \mathrm{C} / \mathrm{cm}^{2}$ [see Fig. 3(b)]. Figure 3(c) shows the overall resistivity modulation of the FeFED obtained upon $P$ reversal. Interestingly, the ratio between the resistivity measured in depletion and accumulation turns out to be $\sim 1.5$ at $300 \mathrm{~K}$ and reaches its maximum of $\sim 3.5$ at $200 \mathrm{~K}$. Moreover, from the depletion to the accumulation state a variation of $\sim 20 \mathrm{~K}$ for the insulator-to-metal transition temperature $\left(T_{\mathrm{IM}}\right)$ is observed as well. Precisely, the latter is varied from the value of $234 \mathrm{~K}$ in depletion to the value of $254 \mathrm{~K}$ in accumulation. The observed variation of $T_{I M}$ is directly linked, through the double-exchange (DE) mechanism, to the modulation of the $T_{\mathrm{C}}$ of LSMO [6]. As the temperature is further decreased, the characteristic metallic behavior of LSMO vanishes for both polarization states. For the LSMO composition used in the present work, insulating and metallic phases are in close competition, therefore phase-separation and/or localization effects may occur, which can be at the origin of the observed upturn of the resistivity for both polarization states at low temperatures.

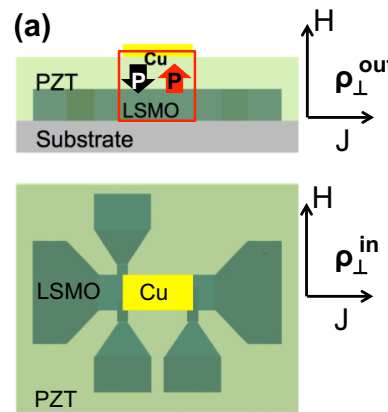

(b)

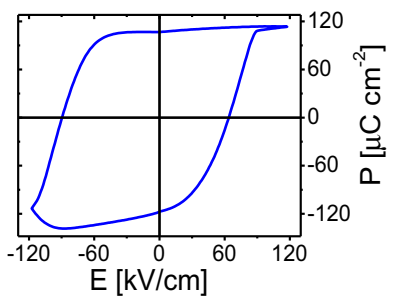

(c)

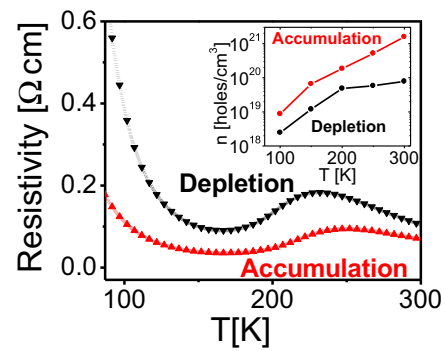

(d)

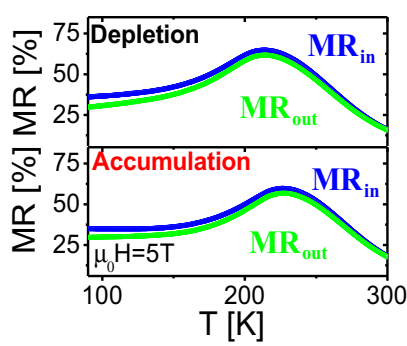

FIG. 3. (Color online) (a) Schematic view of the FeFED used for magnetotransport measurements along with the geometry used to measure the quantities $\rho_{\perp}^{\text {out }}(T)$ and $\rho_{\perp}^{\text {in }}(T)$, respectively. (b) P-E loop of the ferroelectric gate of the investigated FeFED measured in the $\mathrm{Cu} / \mathrm{PZT} / \mathrm{LSMO}$ geometry showing low imprint and stable remanent polarization states. (c) Temperature dependence of the resistivity of the same LSMO layer for both PZT polarization states. The inset shows the charge density modulation achieved upon the $P$ reversal. (d) Magnetoresistance of the LSMO layer acquired with $\mu_{0} H=$ $5 \mathrm{~T}$ oriented perpendicular (green) and parallel (blue) to the FeFED (current) plane for both polarization directions.

Charge density obtained from Hall resistance measurements versus $H$ at several temperatures (not shown here) show a clear modulation of almost one order of magnitude upon reversal of the PZT polarization, demonstrating that the ferroelectric field effect is a genuine electronically driven effect. Figure 3(d) shows the temperature dependence of the magnetoresistance (MR) curves (i.e., $\operatorname{MR}_{\text {out(in) }}=\left[\rho_{\perp}^{\text {out(in) }}(T)-\rho_{H=0}\right] / \rho_{H=0}$; where $\rho_{H=0}$ is the resistivity measured in zero magnetic field), for both accumulation and depletion states, obtained by keeping the $H$ and $J$ vectors always perpendicular to each other. For the MR measurements the amplitude of the magnetic field was chosen to be equal to $5 \mathrm{~T}$. Accordingly, as sketched in Fig. 3(a), $\rho_{\perp}^{\text {out }}(T)$ and $\rho_{\perp}^{\text {in }}(T)$ indicate the resistance measured with $H$ oriented out of plane and in plane with respect to the FeFED plane, respectively. The magnitude of $\mathrm{MR}_{\text {out }}\left(\mathrm{MR}_{\text {in }}\right)$ decreases by polarization switching, driving the LSMO from the depletion to the accumulation state, similar to chemical-doping experiments [21]. Moreover, the distinct difference obtained between $\mathrm{MR}_{\text {out }}$ and $\mathrm{MR}_{\mathrm{in}}$, for both states, indicates an anisotropy of the $\mathrm{MR}_{\text {out }}\left(\mathrm{MR}_{\mathrm{in}}\right)$. Precisely, it is seen that the MR magnitude decreases remarkably when $H$ is oriented perpendicular to the current (FeFED) plane. In this case, the FeFED normal direction ( $c$ axis) represents the hard axis for the field-dependent $\mathrm{MR}_{\text {out }}\left(\mathrm{MR}_{\mathrm{in}}\right)$ measurements. This small but distinct variation of the MR curves by orienting $H$ from the in-plane towards the out-of-plane direction motivated 


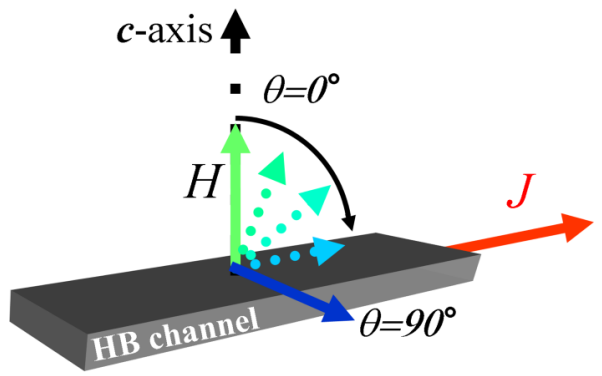

FIG. 4. (Color online) Schematic view of the $H$ rotation with respect to the FeFED plane.

further investigations on the AMR properties of the FeFED channel.

In this respect, isothermal (out of plane) AMR measurements were performed at several temperatures by rotating $H(\perp J)$ from the out-of-plane $\left(\theta=0^{\circ}\right)$ towards the in-plane $\left(\theta=90^{\circ}\right)$ direction, as schematically shown in Fig. 4.

As a result, Fig. 5(a) shows the angular dependence of the resistivity $\rho(\theta)$, exhibiting a two-fold symmetry, measured at $\mathrm{T}=180 \mathrm{~K}$ with $\mu_{0} H=2 \mathrm{~T}$, for both accumulation and depletion states. Figure 5(b) shows the temperature dependence of the normalized AMR amplitude, i.e., $\Delta \rho_{\text {AMR }} / \rho_{H=0}$, with $\Delta \rho_{\text {AMR }}$ being $\rho_{\perp}^{\text {out }}-\rho_{\perp}^{\text {in }}$. The two curves almost overlap at high temperatures but below $T_{\mathrm{C}}(\sim 250 \mathrm{~K})$, a clear splitting occurs and, precisely, a higher value in accumulation than in depletion
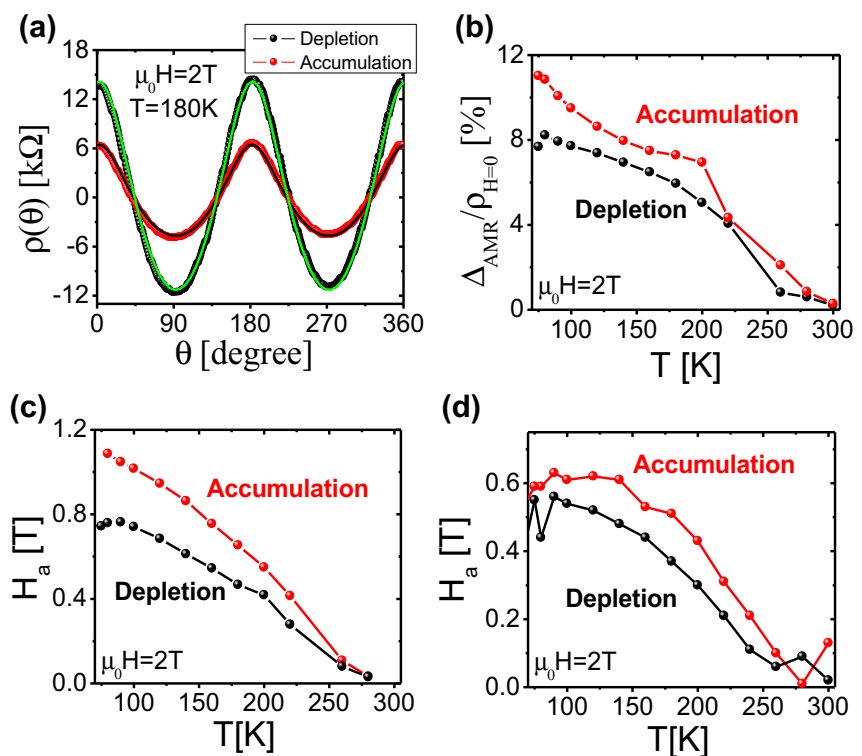

(d)

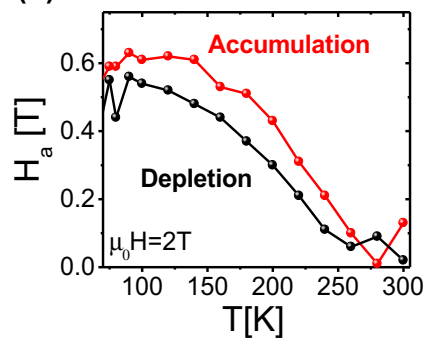

FIG. 5. (Color online) (a) Angular dependence of the resistivity $\rho(\theta)$, exhibiting a two-fold symmetry acquired at the temperature of $180 \mathrm{~K}$ applying a constant magnetic field of $2 \mathrm{~T}$ for both accumulation (red curve) and depletion (black curve) states. The related raw data were simply shifted in order to display the resistivity variation around zero. The solid lines represent a fit made by using the $\cos (2 \phi)$ dependence, $\phi$ being the angle between the device normal and the magnetization. (b), (c) Temperature dependence of the normalized AMR amplitude, i.e., $\Delta \rho_{\mathrm{AMR}} / \rho_{H=0}$ and the anisotropy field, i.e., $H_{a}$, respectively. (d) Temperature dependence of $H_{a}$ related to the FeFED engineered onto a LSAT substrate. is obtained. Remarkably, the latter intriguing electrostatically induced modulation is only related to the out-of-plane AMR, since in the case of the in-plane AMR no difference was found by rotating $H$ with respect to $J$ [22]. Therefore, to gain an insight into the aforementioned modulation, the measured $\rho(\theta)$ curves, all dominated by a uniaxial magnetic anisotropy contribution, were successfully described through the formula $\rho=\rho_{0}+\Delta \rho_{\text {AMR }} \cos (2 \phi)$, where $\phi$ is the angle between $M$ and the $c$ axis, and $\rho_{0}$ is a constant. Accordingly, the fit was performed [see, for example, green and black solid lines in Fig. 5(a)], by calculating $\phi(\theta)$ within the original StonerWohlfarth model [23] (magnetization rotation), considering as magnetic hard axis (due to shape anisotropy) the $c$ axis. As a result, from the fitting procedure (where only the second-order term was considered), the minimum magnetic field value necessary to rotate $M$ out of plane, i.e., the anisotropy field $H_{a}$, was obtained. The latter can be expressed as $\mu_{0} H_{a}=2 K_{\text {tot }} / M_{s}$, where $K_{\text {tot }}$ and $M_{s}$ are the total effective uniaxial anisotropy constant of the system and the saturated magnetization value, respectively. It is important to show that, relative to the magnetoresistance curves acquired as a function of the magnetic field (see Fig. S4 of Supplementary Information [14]), at $H=2 \mathrm{~T}$ the FeFED exhibits, in both depletion and accumulation states, a decreasing trend for $\rho_{\perp}^{\text {out }}(T)\left[\rho_{\perp}^{\text {in }}(T)\right]$ which proves that $M$ can be completely aligned out of plane and, thus, follows the external applied magnetic field for the entire range of temperatures investigated, as already reported in Ref. [24]. Subsequently, at the temperature of $180 \mathrm{~K}, H_{a}$ values of $(0.47 \pm 0.01)$ and $(0.66 \pm 0.01) \mathrm{T}$ were calculated for the accumulation and depletion states, respectively. In this respect, in Fig. 5(c) the calculated $H_{a}$ values are shown as function of the temperature and for both the accumulation and depletion states. Notice that the temperature dependence of the $H_{a}$ values mimics that of the normalized AMR amplitude, and it is observed that, in the accumulation state, a higher magnetic field is required to rotate $M$ out of plane than in depletion. The aforementioned results demonstrate that the electrostatic coupling at the LSMO/PZT interface is capable of inducing a variation of the magnetic anisotropy field as large as $40 \%$ at $180 \mathrm{~K}$. A possible explanation of this mechanism is discussed below.

\section{DISCUSSION}

The magnetic anisotropy of a system consists of shape (magnetostatic), magnetocrystalline, magnetoelastic, and surface anisotropy terms [25]. Correspondingly to the investigated system, the present out-of-plane AMR measurements indicate that the observed two-fold symmetry of the $\rho(\theta)$ curves can be mainly attributed to the shape anisotropy term (which, in the case of thin films, favors an in-plane orientation of $M$ ). In this framework, it emerges that $K_{\text {shape }}=0.5 \mu_{0} M^{2}$ where $K_{\text {shape }}$ is the shape-anisotropy constant and, consequently, it follows that $H_{a}=M_{s} / 2$ (see Ref. [25]). As a result, the same relative percentage variation is expected for $H_{a}$ and $M_{s}$ upon the ferroelectric switching of the FeFED. However, as a matter of fact, we measure (at $180 \mathrm{~K}$ ) $\sim 30 \%$ of variation for $H_{a}$ and only $\sim 6 \%$ for $M_{s}$, on switching from the accumulation to the depletion state. Therefore, one would have to assume that another uniaxial anisotropy contribution term should be responsible 
for the relatively large variation of the $H_{a}$ values. Since the ferroelectric field effect is mainly an interface effect [26], the extra $K_{s}$ term is expected to originate from the broken symmetry that the $\mathrm{Mn}$ ions experience at the LSMO/PZT interface, i.e., the surface magnetic anisotropy term (Néel model [27]). Along this direction, the modulation of the $H_{a}$ values can be argued to be a consequence of the alteration of the energy scale related to the $e_{g}$ levels of the interfacial Mn ions. Indeed, Kyuno and coworkers in Ref. [28] gave a theoretical prediction of the sensibility of the surface magnetic anisotropy of $\mathrm{Ag} / \mathrm{Fe}$ and $\mathrm{Au} / \mathrm{Fe}$ multilayers related to the $3 d$ band filling of the Fe ions. Accordingly, the results obtained from the (magneto) transport characterization of the system established an essential proof that the ferroelectric field effect is capable of electrostatically and reversibly modulating the valence state of the interfacial Mn ions. Interestingly, Chen and coworkers, by using a transmission electron microscopy technique [29], were able to underline the dynamical modulation of the $c / a$ ratio related to the oxygen octahedra surrounding the $\mathrm{Mn}$ ions at the $\operatorname{LSMO}(x=0.2) /$ ferroelectric interface, induced by depletion or accumulation states. Moreover, earlier reports [30] pointed out that substrate-induced strain effects, altering the $c / a$ ratio of the $\mathrm{MnO}_{6}$ octahedra, drive the lowering in energy of either the $3 d z^{2}$ (i.e., compressive strain) or the $3 d x^{2}-$ $y^{2}$ (tensile strain) orbitals which, subsequently, modify the magnetic properties of the system. The latter effect has been also recently proved by Spurgeon and coworkers studying the charge- and strain-mediated effect on the magnetization and, consequently, on the $P$-dependent $3 d$ orbital anisotropy occupancy of a gated LSMO $(x=0.3)$ layer [31]. All these arguments need to propose a valid model that can be used to qualitatively explain the observed modulation of the $H_{a}$ values establishing a link between the ferroelectrically induced displacements of the interfacial Mn and $\mathrm{O}$ ions of the LSMO and the Mn $3 d$ orbital reconstruction (different overlapping and filling). Accordingly, the model is based on the sketch shown in Fig. S5 of Supplementary Information [14], where at the LSMO/PZT interface the depletion (accumulation) state increases (decreases) the $c / a$ ratio, resulting in a preferential out-of-plane $3 d z^{2}$ (in-plane $3 d x^{2}-y^{2}$ ) orbital occupancy. By applying this model to the results shown in Fig. 5(c), a qualitative explanation can be envisaged. Indeed, since in depletion the interfacial $\mathrm{MnO}_{6}$ octahedra expand along the out-of-plane direction, a preferential occupancy of the $3 d z^{2}$ orbitals is obtained. Consequently, the ferromagnetic coupling is enhanced along the out-of-plane direction [32] and the rotation of $M$ is facilitated. As a result, the expected $H_{a}$ values are lower than the one related to the accumulation state where, on the other hand, the out-of-plane shrinking of the $\mathrm{MnO}_{6}$ octahedra triggers a preferential $3 d x^{2}-y^{2}$ orbital occupancy which favors a more stable in-plane orientation of $M$. As a result, the electrostatically induced $\mathrm{Mn} 3 d$ orbital reconstruction at the LSMO/PZT interface accounts for the observed modulation of the $H_{a}$ values. In this respect, the LSMO/PZT interface was uniquely accessible since the rotation of $H$ in a plane perpendicular to $J$, from the hard axis (out-of-plane) towards the easy plane (in-plane) direction, allowed to study the symmetry breaking experienced by the interfacial atoms. To further validate our model an additional experimental way has been used. Specifically, an extrinsic strain-induced out-of-plane elongation of the LSMO unit cell has been obtained by engineering the FeFED onto a different substrate. Precisely, an LSAT substrate with a lattice parameter of $0.387 \mathrm{~nm}$ was used to induce an in-plane compressive strain to the pseudocubic structure of the LSMO (structural and transport properties are given in the Fig. S6 of the Supplementary Information [14]). As a result, since an overall occupancy of the out-of-plane $3 d z^{2}$ orbitals is triggered, a decrease of the $H_{a}$ values, as predicted by the model (superimposed onto the ferroelectric field-effect modulation) is expected. Accordingly, out-of-plane AMR measurements with $H=2 \mathrm{~T}$ have been undertaken, and Fig. 5(d) shows the calculated $H_{a}$ values as a function of the temperature for both accumulation and depletion states. Notice that the $H_{a}$ values, as expected, are remarkably decreased, compared to those obtained on the FeFED grown onto STO. Moreover, the modulation of the $H_{a}$ values between the accumulation and depletion states is still observed, originating from the electrostatical alteration of the interfacial $\mathrm{Mn} 3 d$ orbital occupancy.

In summary, we demonstrated that a ferroelectric polarization is capable of significantly modulating the magnetic and transport properties of a 5-nm-thick LSMO film upon the reversal between its two remanent states. The overall trends observed for the $T_{\mathrm{C}}$ and $T_{\mathrm{IM}}$ temperatures are well explained in the framework of the DE mechanism. Moreover, the capability of the ferroelectric polarization to tune the interfacial magnetic anisotropy of LSMO ultrathin films was demonstrated. The observed variation of the surface magnetic anisotropy is directly linked to a feasible orbital reconstruction, electrostatically induced, that in turn can be responsible for a variation of the SOC at the LSMO/PZT interface. X-ray linear dichroism (XLD) combined with X-ray magnetic circular dichroism (XMCD) measurements, as function of the PZT polarization directions, may elucidate the intrinsic mechanism of the proposed interfacial spin and orbital reconstruction inherent to the Mn ions.

\section{ACKNOWLEDGMENTS}

The authors thank Dr. Marco Salluzzo (University Federico II of Naples, Italy) and Dr. Chengliang Lu (Max Planck Institute, Halle) for fruitful discussions. D.P. thanks the European Community's Seventh Framework Programme (FP7/2007-2013) for financial support under Grant Agreement No. NMP3-LA-2010-246102. I.F. acknowledges Beatriu de Pinós postdoctoral scholarship (2011 BP-A 00220) from the Catalan Agency for Management of University and Research Grants (AGAUR-Generalitat de Catalunya).
[1] E. Dagotto, T. Hotta, and A. Moreo, Phys. Rep. 344, 1 (2001).
[2] K. Chahara, T. Ohno, M. Kasai, and Y. Kozono, Appl. Phys. Lett. 63, 1990 (1993). 
[3] Y. Tokura and Y. Tomioka, J. Magn. Magn. Mater. 200, 1 (1999).

[4] E. Dagotto, Science 309, 257 (2005).

[5] G. H. Jonker and J. H. V. Santen, Physica 16, 337 (1950).

[6] A. Urushibara, Y. Moritomo, T. Arima, A. Asamitsu, G. Kido, and Y. Tokura, Phys. Rev. B 51, 14103 (1995).

[7] C. A. F. Vaz, J. Hoffman, Y. Segal, J. W. Reiner, R. D. Grober, Z. Zhang, C. H. Ahn, and F. J. Walker, Phys. Rev. Lett. 104, 127202 (2010).

[8] H. J. A. Molegraaf, J. Hoffman, and S. Gariglio, Adv. Mater. 21, 3470 (2009).

[9] S. Dong, X. Zhang, R. Yu, and E. Dagotto, Phys. Rev. B 84, 155117 (2011).

[10] E. De Ranieri, A. W. Rushforth, K. Výborný, U. Rana, E. Ahmad, R. P. Campion, C. T. Foxon, B. L. Gallagher, A. C. Irvine, J. Wunderlich, and T. Jungwirth, New J. Phys. 10, 065003 (2008).

[11] R.-W. Li, H. Wang, X. Wang, X. Z. Yu, and Y. Matsui, Proc. Natl. Acad. Sci. USA 106, 14224 (2009).

[12] J. D. Fuhr, M. Granada, L. B. Steren, and B. Alascio, J. Phys.: Condens. Matter 22, 146001 (2010).

[13] A. Asamitsu, Y. Moritomo, Y. Tomioka, T. Arima, and Y. Tokura, Nature (London) 373, 407 (1995).

[14] See Supplemental Material at http://link.aps.org/supplemental/ 10.1103/PhysRevB.90.125155 for extra experimental details.

[15] D. Pesquera, X. Marti, V. Holy, R. Bachelet, G. Herranz, and J. Fontcuberta, Appl. Phys. Lett. 99, 221901 (2011).

[16] J. D. Burton and E. Y. Tsymbal, Phys. Rev. B 80, 174406 (2009).

[17] C.-G. Duan, S. S. Jaswal, and E. Y. Tsymbal, Phys. Rev. Lett. 97, 047201 (2006).

[18] S. Dong and E. Dagotto, Phys. Rev. B 88, 140404 (2013).

[19] L. Jiang, W. Jiang, H. Choi, S. Jeen, Y. Dong, M.-G. Kim, Y. Han, S. Zhu, E. Kalinin, T. Dagotto, H. Egami, and Lee, Nano Lett. 13, 5837 (2013).
[20] I. Vrejoiu, G. L. Rhun, L. Pintilie, D. Hesse, and M. Alexe, Adv. Mater. 18, 1657 (2006).

[21] Y. Tokura, A. Urushibara, Y. Moritomo, T. Arima, and A. Asamitsu, J. Phys. Soc. Jpn. 63, 3931 (1994).

[22] X. Hong, J. D. Hoffman, C. H. Ahn, and Y. Bason, Phys. Rev. B 74, 174406 (2006).

[23] E. C. Stoner and Wohlfarth, IEEE Trans. Magn. 27, 3475 (1991).

[24] J. Eckstein, I. Bozovic, J. O’Donnell, M. Onellion, and M. Rzchowski, Appl. Phys. Lett. 69, 1312, (1996).

[25] B. Cullity and C. Graham, Introduction to Magnetic Materials (Wiley, New York, 2009).

[26] H. Lu, T. A. George, Y. Wang, I. Ketsman, J. D. Burton, C.-W. Bark, S. Ryu, D. J. Kim, J. Wang, C. Binek, P. A. Dowben, A. Sokolov, C.-B. Eom, E. Y. Tsymbal, and A. Gruverman, Appl. Phys. Lett. 100, 232904 (2012).

[27] L. Néel, J. Phys. Radium 15, 255 (1954).

[28] K. Kyuno, J. Ha, R. Yamamoto, and S. Asano, J. Phys. Soc. Jpn. 65, 1334 (1996).

[29] H. Chen, Q. Qiao, M. S. J. Marshall, A. B. Georgescu, A. Gulec, P. J. Phillips, R. F. Klie, F. J. Walker, C. H. Ahn, and S. IsmailBeigi, Nano Lett. 14, 4965 (2014).

[30] A. Tebano, C. Aruta, S. Sanna, P. G. Medaglia, G. Balestrino, A. A. Sidorenko, R. D. Renzi, G. Ghiringhelli, L. Braicovich, V. Bisogni, and N. B. Brookes, Phys. Rev. Lett. 100, 137401 (2008).

[31] S. Spurgeon, J. Spurgeon, D. M. D. Sloppy, P. Kepaptsoglou, S. Balachandran, J. Nejati, A. Karthik, C. Damodaran, H. Johnson, R. Ambaye, V. Goyette, Q. Lauter, J. Ramasse, K. K. S. Idrobo, S. Lau, J. Lofland, L. Rondinelli, M. Martin, and Taheri, ACS Nano 8, 894 (2014).

[32] J. Goodenough, Phys. Rev. 100, 564 (1955). 